\title{
A Novel Secure Interoperation System
}

\author{
Li Jin and Zhengding Lu \\ Department of Computer Science \& Technology, \\ Huazhong University of Science \& Technology, Wuhan 430074, China \\ jessiewelcome@126.com
}

\begin{abstract}
Secure interoperation for a distributed system, such as a multidomain system, is a complex and challenging task. The reason for this is that more and more abnormal requests and hidden intrusions make the static access control policies to be fragile. The access control model presented in this paper approaches this problem by proposing a concept of "trust-level", which involves a statistical learning algorithm, an adaptive calculating algorithm and a self-protecting mechanism to evaluate a dynamic trust degree and realize secure interoperations.
\end{abstract}

Keywords: Secure Interoperation; Trust-level; Adaptive.

\section{Introduction}

Traditional access control systems are defined as granting or denying requests to restrict access to sensitive resources, the main purpose of which is preventing the system from ordinary mistakes or known intrusions. The development of network and distributed technology has made a large revolution in information security area. Sharing information without sacrificing the privacy and security has become an urgent need.

The emergence of Trust Management has promised a novel way to solve these problems. Many researchers have introduced the idea of "trust" to improve a system's security degree. It always involves a system's risk-evaluation or a user's historical reputation to decide whether they are "trust" or not. It is insufficient to deal with such unexpected situations as dishonest network activities, identity spoofing or authentication risk. There are many researchers who have amply discussed the importance of "trust" in a dynamic access control system and reached many achievements. However, how to change an abstract concept of "trust" into a numeric value was insufficiently discussed. The motivation of this paper is drawn by this idea.

To solve these problems, we introduced a new quantitative concept "trust-level" to access control policies and developed a novel Adaptive Secure Interoperation System using Trust-Level (ASITL). In Section 2, we discuss some related achievements of secure interoperation and trust management in recent years. We describe the whole architecture and working flow of the ASITL in Section 3. The trust evaluation module is discussed in Section 4. We also present an interesting example in Section 5. Concluding remarks is added in Section 6. 


\section{Related Works}

Several research efforts have been devoted to the topic of trust strategies in secure interoperations and trust management.

Ninghui Li and John C. Mitchell proposed RT [1], which combines the strengths of Role-based access control and Trust-management systems. RT has been developed into a systematic theory. Some trust services, such as trust establishment, negotiation, agreement and fulfillment were reported in [2], [3]. Although they concluded many security factors that might influence the trust degree, they did not propose a formalized metric to quantize it. Furthermore, Elisa B. et al. discussed secure knowledge management, focusing on confidentiality, trust, and privacy [4] and Kilho S. et al. presented a concrete protocol for anonymous access control that supported compliance to the distributed trust management model [5], both of which represented novel achievements in this area. However, the trust evaluation methods have not been essentially developed.

Comparing with the above research efforts, our framework leads to a number of advantages in a secure interoperation environment.

- A dishonest user can not deceive the authentication server to reach his true intention. Once a dishonest network activity is detected, the user's trust-level will be decreased and he will not be granted any further privileges. Therefore, many potential risks can be efficiently avoided.

- To gain a higher trust-level, a user seeking to any advanced service has to submit correct certificate and obey rules all the time.

- With a statistical learning algorithm, event a new intrusion or an unknown event can also be learned and added into the abnormal events DB.

\section{Framework for ASITL}

ASITL is a self-adaptive secure interoperation system. Different from traditional authentication systems, it involves dynamic trust evaluation mechanism. It consists of three main parts: certificate authentication mechanism, authentication server and trust evaluation module. Each part has its own duty as follows:

Certificate authentication mechanism. It verifies the validity of certificates with the certificates DB.

Authentication server. With the certificate authentication mechanism, access control policies, and if necessary, trust-level evaluation module, it decides whether or not to grant a request of the current user.

Trust evaluation module. This module includes two parts: abnormal judging mechanism and trust-level calculating algorithm. Abnormal judging mechanism involves a self-adaptive statistical learning algorithm which uses a probability method to define a class for an abnormal event. And trust-level calculating mechanism defines a mathematic model to calculate a trust-level value with an abnormal event's kind and its occurrence number. 
With above three main parts, we can describe a typical secure session in ASITL:

Firstly, a user sends a request to the authentication server. And according to user's request and access control policies, the authentication server asks for some necessary certificates.

Secondly, the user submits the needed certificates. If the certificates can satisfy the policies, the user's request will be transmitted to the corresponding application servers. A secure interoperation is finished. Otherwise, the authentication server sends further authentication requirements to the user and trust evaluation module starts to work at once.

Thirdly, the user has to send other certificates once more to further proof his identity. And the authentication server continues to authenticate the current user, at meanwhile, update the trust-level for the user constantly.

Finally, when the user's trust-level is beyond the system's threshold value, the current session will be canceled and the user will be banned in the system for a predefined time-out period. Otherwise, the user has to continue to submit some more certificates to verify his identity until his certificates satisfy the request and access control policies.

\section{Trust Evaluation}

To maintain consistency and simplicity, authentication server generates a user ID and maintains a history record for each user. Generally, a trust observation period is a fixed interval of time, i.e., a threshold defined by system, or a period between two audit-related events.

\subsection{Basic Definitions}

Each network event has it own features. In network security area, researchers often abstractly divide a network event into many significant features. Similarly, common kinds of features can be concluded into a class, which might be related to a certain network event. Before describing the trust evaluation process in detail, we give the basic definitions and theorems.

Definition 1: Every network event contains a set of intrinsic features. When we analysis a network event, some of these features are essential, some of them are irrelevant. We call those essential features as key feature, named feature.

Definition 2: A feature can be divided into a mixture one or more topic kinds, named classes, which are associated with different kinds of network events.

Theorem 1: Supposing that

1. An event $E$ in the abnormal events DB can be described with a feature set $F$;

2. All features $f \in F$ are mutually exclusive and are associated with one or more of a set of classes $C_{k}$;

3. A suspicious event $E_{i}$ is observed by a feature set $F_{J}=\left\{f_{1}, f_{2}, \ldots, f_{j}, \ldots f_{J}\right\}$;

Then the index $I$ of the most probable event $E_{i}$ is given by 


$$
I=\underset{i}{\arg \max } \sum_{J}\left(\log p\left(C_{f(j)} \mid E_{i}\right)-\log p\left(C_{f(j)}\right)\right)
$$

$p(X)$ denotes the probability of event $X$ and $C_{f(j)}$ is the class that feature $f_{j}$ is assigned.

\subsection{Working Flow of Abnormal Judging}

With the definitions and theorems in above, we realize the self-adaptability of abnormal judging mechanism as follows:

Step 1: Initialize the events training set by extracting general features from large amount of abnormal events and learning to deduce some basic rules from current abnormal feature set.

Step 2: Receive an abnormal event which is needed to be classified.

Step 3: Extract the features and send them to the Event Induction module.

(1) If it can be divided into a known class, its abnormal kind will be transferred to the trust-level calculating module.

(2) Otherwise, the unknown features are sent back to the training set and update the current feature rules for next judging process.

\subsection{Trust-Level Calculating}

With determinations made by the abnormal judging module, trust-level calculating algorithm updates the current user's trust-level and feeds back a quantitative "trust" value to the authentication server.

Definition 3: A user's trust-level is defined as follows:

$$
T_{u}=1 / m \underset{k}{\operatorname{Sum}}\left(\alpha_{k}^{l_{k}}\right) \quad(1 \leq k \leq m)
$$

$T_{u}$ denotes the trust-level of user $u$, and $m$ is the amount of abnormal event kinds. $\alpha_{k}$ is the influence rate of each kind of event, which is a real number between 0 and $1 . l_{k}$ is the occurrence number of event $k$. Consequently, $T_{u}$ is in the range of $[0,1] . l_{k}$ starts as 0 to reflect that there is no prior interaction between user and the authentication server (that is, unknown users).

Supposing there are 3 kinds of abnormal event $E_{1}, E_{2}, E_{3}$ and their trust rates are $\alpha_{1}=0.9, \alpha_{2}=0.7, \alpha_{3}=0.5$. The following tables separately shows $T_{u}$ 's going trend as all the kind of events are detected. Assuming that $l_{1}, l_{2}$, and $l_{3}$ all follow the same increasing rate, we can find an interesting result: with the increasing of event kinds, the larger $l_{k}$, the faster the $T_{u}$ decreases. 
Table 1. $T_{u}$ on $E_{1}$ increasing

\begin{tabular}{cccc}
\hline$l_{1}$ & $l_{2}$ & $l_{3}$ & $T_{u}$ \\
\hline 0 & 0 & 0 & 1.0000 \\
3 & 0 & 0 & 0.9097 \\
5 & 0 & 0 & 0.8635 \\
7 & 0 & 0 & 0.8261 \\
9 & 0 & 0 & 0.7958 \\
\hline
\end{tabular}

Table 2. $T_{u}$ on $E_{1}$ and $E_{2}$ increasing

\begin{tabular}{cccc}
\hline$l_{1}$ & $l_{2}$ & $l_{3}$ & $T_{u}$ \\
\hline 0 & 0 & 0 & 1.0000 \\
3 & 3 & 0 & 0.6907 \\
5 & 5 & 0 & 0.5862 \\
7 & 7 & 0 & 0.5202 \\
9 & 9 & 0 & 0.4759 \\
\hline
\end{tabular}

Table 3. $T_{u}$ on $E_{1}, E_{2}$ and $E_{3}$ increasing

\begin{tabular}{cccc}
\hline$l_{1}$ & $l_{2}$ & $l_{3}$ & $T_{u}$ \\
\hline 0 & 0 & 0 & 1.0000 \\
3 & 3 & 3 & 0.3990 \\
5 & 5 & 5 & 0.2633 \\
7 & 7 & 7 & 0.1895 \\
9 & 9 & 9 & 0.1432 \\
\hline
\end{tabular}

\section{An Example}

Assuming there is a file access control system. With the sensitivity $S$ of files, all files can be divided into three classes $\mathrm{A}, \mathrm{B}$, and C $\left(S_{A}>S_{B}>S_{C}\right)$. To maintain secure levels of this system, we defines three different certificates $C_{1}, C_{2}$ and $C_{3}$ $\left(C_{3}>C_{2}>C_{1}\right)$. Different combinations of certificates grant different privileges.

Table 4. File access control policies

\begin{tabular}{cccc}
\hline File & Trust-level & Certificates & History Records \\
\hline A & $0.7 \leq T L<1.0$ & $C_{1}, C_{2}, C_{3}$ & $0.7 \leq A V G_{T L}$ \\
B & $0.4 \leq T L<0.7$ & $C_{1}, C_{2}$ & $0.4 \leq A V G_{T L}$ \\
C & $0.1 \leq T L<0.4$ & $C_{1}$ & $0.1 \leq A V G_{T L}$ \\
\hline
\end{tabular}


Access control policies are defined in Table 4.There are three kinds of abnormal events $E_{1}, E_{2}, E_{3}$ and access control policy defines the lowest threshold of $T_{u}$, named $T_{u T}$, is 0.1000 . Furthermore, $E_{1}, E_{2}, E_{3}$ and their trust rate $\alpha_{1}, \alpha_{2}, \alpha_{3}$ are described as follows:

Certificate_Error_Event: a user presents a needless certificate. Although it is valid, it is not the right one that authentication server needed. This event may indicate a certificate mistake of the user. The trust influence rate of this event is $\alpha_{1}=0.9$.

Certificate_Invalidation_Event: a user presents an expired, damaged or revoked certificate. This event may indicate an attempt to a network fraud. The trust influence rate of it is $\alpha_{2}=0.7$.

Request_Overflow_Event: a user sends abnormally large amounts of requests. This event may indicate an attempt to a Dos attack or a virus intrusion. The trust influence rate of this event is $\alpha_{3}=0.5$.

Jimmy wants to access some files and sends a request with his identity certificate to the authentication server. To demonstrate the secure mechanism of ASITL, we assume three different possible results:

Jimmy is a malicious intruder: He does not have a valid certificate at all. From the beginning, he sends expired or damaged certificates to the authentication server continually. Certificate_Invalidation_Event is detected constantly and the occurrence number of it increases fast. When the occurrence number reaches a threshold amount, Request_Overflow_Event may be detected. Once Jimmy's TL is below 0.1, he will be forbidden by the system. And the final $T L$ with his ID will be recorded in the history record. If this result continually takes place more than five times, the user ID will be recorded in the Black List.

Jimmy is a potentially dangerous user: He only has a valid certificate $C_{1}$, so his privilege only can access the files of Class C. But his true intention is the more sensitive files of Class A or Class B. In order to accumulate a good reputation, he maintains a high $T L(0.4 \leq T L<1.0)$ and $A V G_{T L}\left(0.4 \leq A V G_{T L}\right)$ by validly accessing Class $\mathrm{C}$ files with certificate $C_{1}$. However, once he presents an invalid certificate $C_{2}$ or $C_{3}$, Certificate_Invalidation_Event is trigged and his $T L$ decreases fast. Although Jimmy has owned a high $T L$ and a good history record by dealing with less sensitive files $\mathrm{C}$, his potential intention of more sensitive files $\mathrm{A}$ or $\mathrm{B}$ can never be reached.

Jimmy is a normal user: He sends file request and corresponding valid certificate to the authentication server. If his certificate is suited to the privilege of the request, his $T L$ and history records can satisfy the access control policies, he will pass the authentication and his request will be responded by the application server.

\section{Conclusions and Future Work}

The ASITL, which can supply secure interoperations for multi security domains, is guided by a set of desiderata for achieving a fine-grained access control system. In 
this paper, we introduce a variable value "trust-level" to reflect a user's trust degree. Based on this value, ASITL dynamically evaluates the user's trust degree and responds to the requestors through the judgment of a new suspicious event. Furthermore, ASITL can be sure that all secure measures have been completed before sensitive information is exchanged.

In future work, we would like to extend our work to some new areas. We need find more efficient learning algorithms to shorten the responding period. Neural network algorithms or similar methods might be involved. Moreover, we can further optimize the cooperating abilities among modules in the system to enhance the performance of the system. Finally, trust evaluating for the authentication server and users' privacy issues also need to be investigated.

\section{References}

1. Li, N., Mitchell J., Winsborough W.. RT: A role-based trust-management framework. In: Proceedings of The 3th DARPA Information Survivability Conference and Exposition (DISCEX III), Washington (2003) 201-212

2. Li Xiong, Ling Liu. PeerTrust: Supporting Reputation-Based Trust for Peer-to-Peer Electronic Communities. IEEE Transactions on Knowledge and Data Engineering, Vol.16, No.7 (2004) 843-857

3. Bhavani Thuraisingham,. Trust Management in a Distributed Environment. In: Proceedings of the 29th Annual International Computer Software and Application Conference, vol.2 (2005) 561-572

4. Elisa Bertino, Latifur R. Khan, Ravi Sandhu. Secure Knowledge Management: Confidentiality, Trust, and Privacy. IEEE Transactions on Systems, man, and Cybernetics. Vol. 36, No.3 (2006) 429-438

5. Kilho Shin, Hiroshi Yasuda. Provably Secure Anonymous Access Control for Heterogeneous Trusts. In: Proceedings of the First International Conference on Availability, Reliability and Security (2006) 24-33 